\title{
MUDANÇAS NO CONSUMO ALIMENTAR E NAS CONDIÇÕES PSICOSSOCIAIS GERADAS PELA DOENÇA INFLAMATÓRIA INTESTINAL
}

\author{
CHANGES IN FOOD CONSUMPTION AND \\ PSYCHOSOCIAL CONDITIONS GENERATED BY \\ INFLAMMATORY BOWEL DISEASE
}

\author{
Thaise Morgana Batista ${ }^{1}$ \\ Claiza Barretta ${ }^{2}$ \\ Cristina Henschel de Matos ${ }^{3}$ \\ Sueli Terezinha Bobato ${ }^{4}$ \\ Everson Fernando Malluta ${ }^{5}$ \\ Bruno Lorenzo Scolaro ${ }^{6}$ \\ Munique Kurtz de Mello ${ }^{7}$ \\ Clarice Maria Specht ${ }^{8}$
}

RESUMO: As Doenças Inflamatórias Intestinais (DII) são distúrbios inflamatórios intestinais crônicos, cuja atividade gera impactos em vários âmbitos da vida do paciente, como no consumo alimentar e no estado psicológico. O presente trabalho avaliou as mudanças no consumo alimentar e nas condições psicossociais geradas pelo desenvolvimento da DII. Foi realizado um estudo transversal e descritivo, por meio da aplicação de um formulário aos pacientes assistidos em um Ambulatório Interdisciplinar do município de Itajaí - Santa Catarina, contendo perguntas abertas e fechadas, sobre o consumo de alimentos, as restrições alimentares e as alterações psicossociais que possam ter sido gerados pela doença em decorrência dos sintomas. Dos 65 pacientes avaliados, 90,8\% restringem algum alimento/bebida, sendo os mais evitados: álcool, refrigerante, leite e derivados e alimentos gordurosos; e 89,2\% sofreram alguma modificação psicossocial após o desenvolvimento da doença. Diante disto, as mudanças alimentares e psicossociais parecem iminentes ao diagnóstico de DII. A doença ainda parece ter gerado impacto em âmbitos socioemocional e profissional na vida desses pacientes. Dessa forma, faz-se necessário um atendimento interdisciplinar, além de um olhar humanizado e integral por parte dos profissionais para esse paciente, que perpassa por modificações muito além das biológicas.

PALAVRAS-CHAVE: Colite Ulcerativa. Doença de Crohn. Consumo Alimentar. Impacto Psicossocial.

Licença CC BY: Artigo distribuído sob os termos Creative Commons, permite uso e distribuição irrestrita em qualquer meio desde que o autor credite a fonte original.
Acadêmica do Curso de Nutrição da Universidade do Vale do Itajaí - UNIVALI.

2 Nutricionista. Mestre em Ciências Farmacêuticas pela Universidade do Vale do Itajaí - UNIVALI. Professora titular da UNIVALI. E-mail: claizabarretta@yahoo.com.br.

3 Nutricionista. Mestre em Engenharia de Produção pela Universidade Federal de Santa Catarina - UFSC. Professora titular da UNIVALI.E-mail: cmatos@univali.br.

4 Psicóloga. Mestre em Psicologia pela Universidade Federal de Santa Catarina UFSC.E-mail: suelibobato@univali.br.

5 Médico. Doutor em Medicina pela Universidade de São Paulo - USP.

6 Médico. Mestre em Saúde e Meio Ambiente pela Universidade da Região de Joinville - UNIVILLE.

7 Médica. Especialista em Saúde Nutricional Integral pelo GANEP Nutrição Humana.

8 Enfermeira Obstétrica. Mestre em Saúde e Gestão do Trabalho pela Universidade do Vale do Itajaí - UNIVALI. Professora titular da UNIVALI.E-mail: cms@univali.br. 


\begin{abstract}
Inflammatory Bowel Diseases are chronic intestinal inflammatory disorders, whose activity generates impacts in several areas of the patient's life, such as food consumption and psychological status. The present study evaluates the changes in food consumption and psychosocial conditions generated by the development of Inflammatory Bowel Disease. A cross-sectional and descriptive study was carried out, through the application of a form for patients attending an Interdisciplinary Outpatient Clinic in the city of Itajaí - Santa Catarina, with open and closed questions about food consumption, dietary restrictions and psychosocial changes that may have been caused by the disease, as a result of the symptoms. Of the 65 patients evaluated, $90.8 \%$ restricted some food or drink, with most avoiding alcohol, soda, milk and derivatives, and fatty foods; and $89.2 \%$ suffered some psychosocial change after the development of disease. In view of this, dietary and psychosocial changes seem imminent to the diagnosis of IBD. The disease still seems to generate socioemotional and professional impacts on the lives of these patients. An interdisciplinary care is therefore needed, together with a humanized and integral look by health professionals, at this patient, whose symptoms go far beyond biological ones.
\end{abstract}

KEYWORDS: Ulcerative Colitis. Crohn Disease. Food Consumption. Psychosocial Impact.

\title{
1 INTRODUÇÃO
}

As Doenças Inflamatórias Intestinais (DII) são distúrbios inflamatórios intestinais crônicos, desencadeados por uma resposta imune inapropriada. Entre as principais doenças desse grupo estão a Retocolite Ulcerativa Idiopática (RCUI) e a Doença de Crohn (DC) ${ }^{3,6}$.

As características genéticas, epidemiológicas e imunológicas são semelhantes entre a RCUI e a DC, no entanto existem diferenças importantes na forma de apresentação. Na RCUI a resposta inflamatória e as alterações morfológicas são restritas ao cólon e ao reto, com comprometimento contínuo e delimitado à mucosa. Enquanto a DC é um processo inflamatório transmural que pode afetar todo o trato gastrointestinal de maneira descontínua. Ambas têm como característica a alternância de episódios de atividade e remissão, e extensão variáveis ${ }^{3,13,15}$.

A etiologia dessas doenças permanece desconhecida, embora se acredite que estejam envolvidos fatores ambientais, genéticos, imunológicos, infecciosos e psicológicos. Entretanto, o aumento da incidência aponta que os fatores ambientais desempenham um papel significativo para o desenvolvimento das DII, contudo não é bem compreendido de que forma esses fatores iniciam ou reativam a doença ${ }^{3,10}$.

A dieta contribui para a patogênese da DII como um fator ambiental. Embora na fase de remissão o estado nutricional seja aparentemente normal, os pacientes com DII ativa são mais propensos à desnutrição com perda de peso e deficiências específicas de nutrientes, especialmente pela redução na ingestão de alimentos, má absorção, pelo aumento das necessidades nutricionais e perdas gastrointestinais ${ }^{8,22,27}$.

Os fatores psicológicos também se destacam no curso da doença, visto que existe forte indício do impacto das emoções sobre as DII. É estabelecido que determinadas condições psicológicas, como ansiedade e depressão, decorrentes de sintomas e estigmas dessas doenças, influenciam a motilidade gastrointestinal e a resposta imunológica com maior propensão durante a atividade inflamatória. Dessa forma, aspectos emocionais poderiam agravar os sintomas ou provocar recidivas. Além disso, as DII comprometem a qualidade e a expectativa de vida dos pacientes e representam um impacto social e psicológico importante $e^{8,9,15,18}$.

Embora haja poucos dados epidemiológicos sobre essas afecções no Brasil, a prevalência de DII é considerada baixa no país. Entretanto, relatos na literatura brasileira mostram um aumento 
significativo dessas doenças e, para alguns autores, as DII podem estar sendo subdiagnosticadas por não serem de notificação compulsória ${ }^{5,10,17,29}$.

De forma geral, há muitas controvérsias e poucos estudos relacionando ao possível papel desempenhado pelos aspectos nutricionais e psicológicos no desenvolvimento e/ou curso clínico da DII ${ }^{18}$. Diante disso, o objetivo deste trabalho foi avaliar mudanças no consumo alimentar e nas condições psicossociais geradas pelo desenvolvimento da Doença Inflamatória Intestinal.

\section{MÉTODOS}

Trata-se de um estudo transversal e analítico, cujos critérios de inclusão foram pacientes com diagnóstico de DII, maiores de 18 anos, atendidos no Ambulatório Interdisciplinar de DII da Unidade de Saúde Familiar e Comunitária (USFC) da Universidade do Vale do Itajaí (UNIVALI) em Itajaí, SC, Brasil. O ambulatório Interdisciplinar de DII é um projeto que tem por objetivo unir ensino, pesquisa e extensão na assistência multidisciplinar e interdisciplinar aos pacientes portadores de doenças inflamatórias intestinais, encaminhados pelas unidades básicas de saúde e hospitalares de cidades pertencentes à Associação dos Municípios da Foz do Rio Itajaí (AMFRI) e seus familiares. Surgiu em 2011 a partir de uma parceria entre os Cursos de Medicina, Nutrição, Enfermagem, Psicologia e a Unidade de Saúde Familiar e Comunitária. Consiste no trabalho de assistência por meio de consultas médicas, nutricionais e psicológicas semanais e administração de medicamentos. Ao todo estão cadastrados no ambulatório 140 pacientes e são realizadas 40 consultas por mês. Após a aplicação dos critérios de inclusão e exclusão, foi constituída uma amostra por demanda espontânea de 65 pacientes. A coleta de dados foi iniciada após a aprovação pelo Comitê de Ética em Pesquisa, sob o parecer número 1.868.893 e ocorreu entre o período de março a agosto de 2017. Os pacientes foram esclarecidos sobre os objetivos da pesquisa e posteriormente assinaram o Termo de Consentimento Livre e Esclarecido.

O formulário foi aplicado pelos pesquisadores na sala de espera do ambulatório interdisciplinar de DII. Foram avaliados dados sociodemográficos a partir das seguintes variáveis: idade, sexo (feminino e masculino) e estado civil (solteiro, casado, união estável, viúvo, separado); dados socioeconômicos: escolaridade (analfabeto, fundamental incompleto ou completo; médio incompleto ou completo e superior incompleto ou completo); renda familiar (até 2 salários mínimos (SM); 3-5 SM; 6-10 SM; 11 - 20 SM; acima de 20 SM); dados da doença: tipo (DC; RCUI); e atividade da doença (remissão; em atividade). Ainda, perguntas abertas e fechadas, com respostas dicotômicas e de única ou múltipla escolha, sobre o consumo e restrições alimentares, sentimentos relacionados às modificações pós-diagnósticas, bem como reflexos dessas mudanças nas dimensões psicossociais dos participantes. As perguntas que abordavam questões psicossociais e alimentares foram elaboradas com base no estudo de Norton et al. ${ }^{20}$ que avaliaram o impacto da DC em aspectos da vida diária.

Para a avaliação antropométrica, o peso foi aferido em balança digital com capacidade máxima de $180 \mathrm{~kg}$ e precisão de $0,1 \mathrm{~kg}$, sem casacos ou agasalhos e descalços, e a estatura mensurada em estadiômetro acoplado a uma balança mecânica, com escala de $0,1 \mathrm{~cm}$, sem sapatos, posição ereta, calcanhares juntos e de costas para a balança. O Índice de Massa Corporal (IMC) foi calculado conforme a fórmula: $\mathrm{IMC}=\operatorname{Peso}(\mathrm{kg}) /$ Altura $^{2}(\mathrm{~m})$. Para a classificação do estado nutricional foram utilizados os critérios propostos pela World Health Organization ${ }^{31}$ para adultos e pela Nutrition Screening Initiative $e^{21}$ para idosos. Para fins estatísticos, a classificação de IMC foi categorizada em baixo peso, eutrofia e excesso de peso (sobrepeso/obesidade). 
Para verificar a atividade inflamatória da DC, foi aplicado o índice clínico de atividade proposto por Harvey-Bradshaw que avalia a sensação de bem-estar geral, dor abdominal, número de evacuações líquidas diárias, presença de massa abdominal e complicações decorrentes da DC ${ }^{16 .}$ Os pacientes foram considerados em fase de remissão quando obtiveram pontuações $<5$, em atividade se pontuação $\geq 5$. Para os pacientes diagnosticados com RCUI, o índice utilizado foi o Escore Mayo Parcial, que avalia o número de evacuações, sangramento retal e avaliação global ${ }^{26}$, sendo considerados em remissão os pacientes que atingissem pontuação $<2$, e em atividade se pontuação $\geq 2$.

Os dados coletados na pesquisa foram tabulados com os programas Microsoft Excel ${ }^{\mathbb{B}}$ e $W_{o r d}{ }^{\mathbb{R}}$ e a análise estatística foi realizada com auxílio do programa $S T A T A 13.0^{\circledR}$. Para as variáveis categóricas, foram calculadas as frequências absolutas e relativas (número e \%) e as associações analisadas pelo teste $\chi^{2}$ ou teste exato de Fischer. Para as variáveis quantitativas, foi calculada média (mínimo e máximo) e as correlações foram feitas pelo teste de Spearman.

\section{RESULTADOS E DISCUSSÃO}

\subsection{AspeCtOS GERAIS E ESTADO NUTRICIONAL}

Foram entrevistados 65 pacientes com DII no período de 03 de março a 04 de agosto de 2017. Todos eram alfabetizados e 96,9\% $(\mathrm{n}=63)$ procediam de cidades do Vale do Itajaí; 72,3\% $(\mathrm{n}=47)$ eram do sexo feminino e a média de idade geral foi de 49,7 $\pm 14,08$ anos. Eram casados ou viviam em união estável $64,6 \%(n=42)$ dos participantes. O tempo de diagnóstico variou de 6 meses a 27 anos. Apenas 13,8\% $(\mathrm{n}=9)$ possuíam histórico familiar de DII. Já passaram por internação hospitalar 53,8\% $(\mathrm{n}=35)$ e fizeram alguma cirurgia pela doença 18,5\% (n=12). Quanto à atividade da doença, 30,8\% (n=20) apresentavam algum grau de atividade, classificada pelos índices de Harvey e Bradshaw e Escore Mayo Parcial. Mais da metade (56,9\%; n = 37) possuía excesso de peso (sobrepeso/obesidade), 36,9\% $(\mathrm{n}=24)$ estavam eutróficos e apenas 6,2\% $(\mathrm{n}=4)$ com baixo peso; o IMC médio foi de $25,91 \pm 5,03 \mathrm{~kg} / \mathrm{m}^{2}$ (Tabela 1 ).

Tabela 01: Caracterização da população e indicador antropométrico de pacientes atendidos no Ambulatório de DII, Itajaí - SC, 2017.

\begin{tabular}{|l|c|c|c|}
\hline & DC & RCUI & TOTAL \\
\hline População (homem; mulher) & $36(11 ; 25)$ & $29(7 ; 22)$ & $65(18 ; 47)$ \\
\hline Idade média em anos (mín.; máx.) & $50,8(21 ; 75)$ & $48,4(21 ; 75)$ & $49,7(21 ; 75)$ \\
\hline Tempo médio de diagnóstico em anos (min.; máx.) & $7,8(1 ; 27)$ & $7,5(0,5 ; 23)$ & $7,7(0,5 ; 27)$ \\
\hline Remissão da doença [n (\%)] & $24(36,9)$ & $21(32,3)$ & $45(69,2)$ \\
\hline Atividade da doença [n (\%)] & $12(18,5)$ & $8(12,3)$ & $20(30,8)$ \\
\hline IMC médio \pm DP $\left(\mathrm{kg} / \mathrm{m}^{2}\right)$ & $26,05 \pm 5,67$ & $25,74 \pm 4,19$ & $25,91 \pm 5,03$ \\
\hline
\end{tabular}

O tratamento dietético é importante para as DII e tem por objetivo recuperar e/ou manter o estado nutricional, fornecer aporte adequado de nutrientes, aliviar sintomas e diminuir a atividade da doença ${ }^{23}$. No entanto, quando avaliada a relação entre o estado nutricional e a atividade da doença $(\mathrm{p}=0,572)$, não houve associação estatisticamente significativa neste estudo. 
Para Silva et al. ${ }^{28}$, pacientes acompanhados em ambulatório tendem a ter um estado nutricional preservado devido ao monitoramento e à orientação alimentar durante o tratamento. Semelhante ao presente estudo, os autores encontraram maior número de excesso de peso (85\%) ao avaliarem 55 pacientes com DII em diferentes estágios de inflamação, e apesar de também não terem identificado associação estatística entre o estado nutricional e a atividade da doença, os pacientes com excesso de peso e gordura corporal eram mais sintomáticos e apresentavam maiores valores de proteína $\mathrm{C}$ reativa, marcador inflamatório associado à atividade clínica da doença ${ }^{28}$.

A prevalência de sobrepeso e de obesidade tem aumentado entre os pacientes com DII nas últimas décadas, e está associada ao aumento do risco de complicações, como maior número de recidivas da doença e hospitalizações mais frequentes. A avaliação nutricional pode auxiliar na identificação de deficiências ou excessos nutricionais e é um ponto importante para o sucesso no manejo de pacientes com DII ${ }^{4,7}$.

\subsection{Aspectos psicossociais}

Como mostra a Tabela 2, a maioria dos pacientes respondeu ter sofrido algum constrangimento após o desenvolvimento da doença, seja pelas idas frequentes ao banheiro ou pela flatulência excessiva, e muitos deixaram de ir a algum local (eventos sociais, festas de aniversário, casamento, igreja e restaurantes) em função dos sintomas gerados pela DII. Alguns pacientes disseram que não iam a lugar nenhum quando descobriram a doença ou que quase não saíam quando estavam em crise, outros já deixaram de viajar, de sair com a família ou amigos, de ir à casa de amigos ou parentes, a estádios de futebol ou não iam a locais que não tivessem banheiro.

Tabela 02: Experiências e sentimentos gerados pela doença de pacientes atendidos no Ambulatório de DII, Itajaí - SC, 2017.

\begin{tabular}{|l|c|c|c|c|}
\hline \multirow{2}{*}{ PERGUNTA } & \multicolumn{2}{|c|}{ SIM } & \multicolumn{2}{|c|}{ NÃO } \\
\cline { 2 - 5 } & $(\mathrm{n})$ & $(\%)$ & $(\mathrm{n})$ & $(\%)$ \\
\hline As idas ao banheiro já lhe causaram/causam constrangimento? & 43 & 66,2 & 22 & 33,8 \\
\hline A flatulência excessiva já lhe causou/causa constrangimento? & 32 & 49,2 & 33 & 50,8 \\
\hline Você já deixou/deixa de ir a algum lugar por causa dos sintomas? & 42 & 64,6 & 23 & 35,4 \\
\hline Já precisou faltar ou se afastar do trabalho pela doença? & 37 & 56,9 & 28 & 43,1 \\
\hline Você já mudou de emprego/saiu do mercado de trabalho pela doença? & 19 & 29,2 & 46 & 70,8 \\
\hline Você tem algum medo em relação à doença? & 33 & 50,8 & 32 & 49,2 \\
\hline Realiza acompanhamento psicológico? & 15 & 23,1 & 50 & 76,9 \\
\hline
\end{tabular}

Norton et al. ${ }^{20}$ já haviam descrito situações semelhantes após avaliarem o impacto da DC em aspectos da vida diária a partir da perspectiva de 48 pacientes que vivem com DC moderada/ grave nos Estados Unidos da América (EUA). Segundo os autores, a DII gerou impacto psicológico na vida dos pacientes por ser vista como silenciosa e constrangedora, em decorrência de idas frequentes ao banheiro, medo de sujar-se ou pela flatulência excessiva. Ainda foram relatados: impacto social, por autoexclusão social ou preocupação sobre viajar longas distâncias, em decorrência dos sintomas, idas ao banheiro ou dieta; e impacto na vida profissional, por redução de horas trabalhadas, mudança de carreira ou saída do mercado de trabalho. 
No presente estudo, mais da metade dos pacientes avaliados já precisou faltar ou se afastar do trabalho em algum momento, e quase um terço mudou de emprego ou parou de trabalhar em decorrência da doença (Tabela 2). Além disso, alguns relatos sugerem o impacto da doença na vida profissional: um dos pacientes referiu se sentir mal com as faltas e as saídas antecipadas, por saber que seu chefe "não gosta"; uma mulher alegou ter sofrido um acidente de trânsito, gerado pelo estresse ao ter perdido o emprego; outro paciente se aposentou por invalidez, devido à colocação de bolsa coletora; e dois outros referiram ter se afastado, em decorrência da ocupação que tinham, uma por trabalhar como manipuladora de alimentos e os sintomas gastrointestinais colocarem em risco a segurança do alimento; e o outro por ser pescador, já que os sintomas lhe impossibilitavam de permanecer em alto-mar. Para Hardt et al. ${ }^{15}$, o impacto no trabalho também se dá pelo distúrbio ocorrer principalmente entre adolescentes e adultos jovens, grupo etário de grande produtividade.

Ao serem questionados sobre ter algum medo em relação à doença, 50,8\% responderam afirmativamente (Tabela 2). Os maiores medos foram de que a doença agrave/evolua para câncer $(30,8 \% ; \mathrm{n}=20)$, seguido dos medos de precisar de cirurgia ou de bolsa coletora $(9,2 \% ; \mathrm{n}=6)$ e de morrer $(7,7 \% ; \mathrm{n}=5)$; ainda foram citados os medos de engravidar $(1,5 \% ; \mathrm{n}=1)$ e de não poder ter filho $(1,5 \% ; \mathrm{n}=1)$. Algumas mulheres avaliadas por Norton et al. ${ }^{20}$ também citaram o medo de engravidar e sentir-se mal durante a gravidez. Uma paciente do presente estudo relatou ter tido problemas conjugais por querer engravidar e seu marido não concordar por medo de sua morte, já que seria uma gestação de risco por ela ser “doente”. Além disso, pacientes com DC parecem ter maiores taxas de infertilidade, quando comparadas a controles gerais ${ }^{24}$.

De fato a imprevisibilidade, a incerteza e o curso crônico da doença inflamatória intestinal podem causar preocupações psicológicas e interpessoais para os pacientes, e para tanto são necessários enfrentamento apropriado, estratégias e boa adaptação. O estresse psicológico é um fator patogênico na DII, dessa forma uma intervenção psicológica pode ser destinada à redução do estresse e potencialmente à atividade da doença. Além disso, as intervenções psicológicas podem melhorar a capacidade de o paciente lidar e gerenciar sua angústia ${ }^{25}$. A vulnerabilidade emocional e a necessidade de acompanhamento psicológico de pacientes com DII também foram abordadas por outros autores ${ }^{1,9,14}$. No presente estudo, 23, $1 \%$ dos pacientes realizavam acompanhamento psicológico (Tabela 2 ).

Ainda sobre os sentimentos causados pela DII, os pacientes foram questionados sobre o que sentiram quando receberam o diagnóstico e como se sentem atualmente. Quinze pacientes $(23,1 \%)$ responderam tristeza; 13 (20\%) nada sentiram; $10(15,4 \%)$ responderam alívio; 8 (12,3\%) sentiram dúvidas ou medo; outros $(16,9 \% ; \mathrm{n}=11)$, frustração, raiva, insegurança, preocupação ou surpresa. Uma paciente referiu ter tido depressão devido ao diagnóstico e outra que achou que "seu mundo fosse acabar". No dia em que foram entrevistados, a maioria $(58,5 \% ; \mathrm{n}=38)$ sentia-se conformada com a doença; e 4,6\% (n = 3) disseram sentir-se bem; o restante $(36,9 \%$; $n$ = 24) dividiu-se entre os sentimentos de confusão, frustração, indiferença, tristeza, insegurança, medo, culpa, preocupação e incômodo. Não houve associação estatística entre a atual relação com o diagnóstico e a realização de acompanhamento psicológico $(p=0,492)$. Entretanto, aceitar a doença pode contribuir para a manutenção do estado remissivo, pois de acordo com Gouveia e Ávila ${ }^{14}$, embora os fatores emocionais não possam ser considerados causadores diretos da doença, devem ser vistos como "elementos precipitadores, contribuidores e/ou exacerbadores". Para os autores, o tratamento físico das doenças psicossomáticas é de fundamental importância, mas o tratamento psicoterapêutico também deve auxiliar nesse segmento para reorganização do processo corporal ${ }^{14}$. 


\subsection{MudanÇAS PSICOSSOCIAIS}

As principais mudanças psicossociais ocorridas na vida dos pacientes após o diagnóstico também foram questionadas e 89,2\% ( $\mathrm{n}=58)$ dos avaliados referiram ter sofrido alguma modificação. Dentre as opções, a mais escolhida foi alteração de humor (Tabela 3). Lima et al. ${ }^{18}$ já haviam encontrado resultado semelhante ao estudar a oscilação do humor, presente na maioria dos pacientes (58\%) com DC de um ambulatório de DII de Juiz de Fora.

Tabela 03: Modificações psicossociais de pacientes atendidos no Ambulatório de DII, Itajaí - SC, 2017.

\begin{tabular}{|l|c|c|}
\hline MODIFICAÇÕES PSICOSSOCIAIS & (n) & (\%) \\
\hline Alteração de humor & 27 & 41,5 \\
\hline Dificuldade financeira & 18 & 27,7 \\
\hline Aumento da dependência de outros & 17 & 26,2 \\
\hline Diminuição da libido & 14 & 21,5 \\
\hline Redução de horas trabalhadas & 12 & 18,5 \\
\hline Hábitos de vida & 11 & 16,9 \\
\hline Perda do emprego & 10 & 15,4 \\
\hline Não houve modificações & 7 & 10,8 \\
\hline Mudança de carreira & 5 & 7,7 \\
\hline
\end{tabular}

Outra modificação que se destacou entre os participantes foi a dificuldade financeira, que pode ter sido gerada pelas mudanças profissionais ocorridas, como a perda do emprego, a redução de horas trabalhadas ou a mudança de carreira (Tabela 3). Para 3 pacientes (4,6\%), os problemas financeiros foram agravados pela falta de medicamentos excepcionais (de uso contínuo e alto custo) do Sistema Único de Saúde (SUS). Vale destacar que 47,7\% (n=31) dos pacientes não trabalham ou são aposentados e 50,8\% $(\mathrm{n}=33)$ possui baixa renda familiar (até 2 salários mínimos). Para Sainsbury e Heatley ${ }^{24}$, pacientes de classes sociais inferiores têm menor qualidade de vida e maior morbidade, por serem propensos a não buscar cuidados de saúde.

A DII pode ainda gerar problemas de autoestima, de intimidade e perda do desejo sexual ${ }^{20}$. Neste estudo, a diminuição de libido ocorreu com 21,5\% dos entrevistados (Tabela 3). Timmer et al. ${ }^{30}$ avaliaram determinantes da função sexual feminina de 336 pacientes com DII e encontraram $63 \%$ de diminuição da atividade sexual, entretanto associaram a fatores psicossociais e não a características específicas relacionadas à doença; enquanto para Alonso, San Román e Miquel², a atividade da doença também é um fator que influencia a sexualidade dos pacientes, bem como o humor depressivo e a presença de doenças concomitantes.

O aumento da dependência de outros e a mudança de hábitos de vida como beber ou fumar também foram citados (Tabela 3). No entanto, o tabagismo, de acordo com Faust et al. ${ }^{11}$, está associado à diminuição da qualidade de vida desses pacientes, além de ser um fator de risco para o desenvolvimento e a exacerbação da $\mathrm{DC}^{24}$. Faveri et al. ${ }^{12}$ já haviam sugerido que a presença da doença pode modificar hábitos de vida ao identificar 15\% de redução de tabagismo em pacientes que receberam o diagnóstico de DII.

Apesar de todas as modificações psicossociais que a doença gerou, alguns $(20 \%$; $=$ 13) consideraram as mudanças favoráveis, pois referem que melhoraram a qualidade de vida após o diagnóstico. 
Quando questionados sobre o que mais incomoda na doença, 41,5\% (n = 27) afirmaram ser os sintomas; $21,5 \%(\mathrm{n}=14)$, as restrições alimentares; $13,9 \%(\mathrm{n}=9)$ não se incomodam; 12,3\% (n = 8) não gostam do constrangimento causado; e 4,6\% $(\mathrm{n}=3)$, por outros motivos, como a doença não ter cura, a quantidade de medicamentos utilizada e, para uma paciente, não saber "como será seu fim” (entende-se futuro). A falta de conhecimento social sobre a doença também é considerada desfavorável para alguns $(6,2 \%$; $=4)$; uma das entrevistadas referiu que precisou utilizar o banheiro de uma loja comercial e não a deixaram, gerando desconforto e medo de não conseguir segurar-se até encontrar outro local. Além de situações como essa, o pouco conhecimento e a falta de compreensão da sociedade ou de familiares sobre o assunto podem promover sentimentos de depressão e solidão ${ }^{20}$.

Contudo, a mudança mais representativa parece ser a alimentar, já que 90,8\% ( $\mathrm{n}=59)$ dos pacientes evitam comer/beber algo pela doença; e 40\% $(n=26)$ já deixou de ir a algum evento social em função das restrições alimentares. Um avaliado referiu não deixar de sair pelas restrições, no entanto se sente incomodado ao ter que dar explicações às pessoas por não poder comer.

\subsection{MudanÇAS ALimentares}

Foi questionado aos pacientes quais eram os alimentos/bebidas evitadas por eles, por que eram e como eles se sentiam em relação à restrição. As restrições citadas em maior número foram bebidas alcoólicas, refrigerante, leite e derivados e alimentos gordurosos (Tabela 4).

Tabela 04: Alimentos e bebidas evitados pelos pacientes atendidos no Ambulatório de DII, Itajaí - SC, 2017.

\begin{tabular}{|l|c|}
\hline ALIMENTOS / BEBIDAS & $\begin{array}{c}\text { NÚMERO DE } \\
\text { CITAÇÕES }\end{array}$ \\
\hline $\begin{array}{l}\text { Bebidas industrializadas, com cafeína e álcool (café, cerveja, destilados, refrigerante, } \\
\text { suco e vinho) }\end{array}$ & 34 \\
\hline Leite e derivados (iogurte, lactose, nata, requeijão e queijo) & 33 \\
\hline Frituras e alimentos gordurosos & 28 \\
\hline $\begin{array}{l}\text { Carnes bovina, suína, de aves e frutos do mar (camarão, lula, marisco, polvo e alguns } \\
\text { tipos de peixe, como atum) }\end{array}$ & 15 \\
\hline Doces (açúcar, bolo, chocolate e sorvete) & 12 \\
\hline Feijão, feijoada e lentilha & 12 \\
\hline Alimentos industrializados e embutidos (presunto, salame, salgadinho e salsicha) & 11 \\
\hline Alimentos com glúten (macarrão, pão branco e pizza) & 11 \\
\hline Condimentos (conservas, molhos, pimenta e temperos) & 10 \\
\hline Frutas ácidas, alimentos ácidos e verduras (couve, couve-flor, laranja e repolho) & 9 \\
\hline Outros (amendoim, fibras, milho, pipoca, polenta) & \\
\hline
\end{tabular}

Corroborando os dados achados no presente estudo, Faveri et al. ${ }^{12}$ também observaram redução no consumo de refrigerante, café, frutas e hortaliças nesta mesma população. Além disso, os autores identificaram correlação positiva e significativa entre o consumo de frutas e hortaliças e o estado nutricional após o diagnóstico da doença. 
Ripoli et al. ${ }^{22}$ encontraram redução no consumo de todos os grupos de alimentos, como cereais, legumes, óleos e gorduras, e de nutrientes como carboidratos, proteínas, lipídios, cálcio, ferro e fósforo em 37\% dos pacientes com RCUI ativa, de um ambulatório de gastroenterologia em $\mathrm{SP}$, onde foram acompanhados 65 pacientes.

Embora a grande maioria dos pacientes deste estudo estivesse eutrófica ou com excesso de peso, não é possível afirmar que não possuía alguma deficiência de nutrientes, já que 90,8\% deles evitam comer/beber algo pela doença, e a diminuição da ingestão e a má absorção contribuem para o comprometimento do estado nutricional ${ }^{27}$.

Estudos mostram que a diminuição da ingestão alimentar está relacionada a sintomas como anorexia, inapetência, náusea, vômito, diarreia, dieta restrita e também à ansiedade e ao medo de comer, decorrentes de experiências de dor e distensão abdominal ${ }^{13,27}$. Os motivos descritos pelos pacientes desse estudo foram: a) causam dor, desconforto ou distensão abdominal; b) para evitar reativação da doença, sintomas ou piorá-los; c) se sentem mal ao consumir; d) causam diarreia; e) por orientação médica/nutricional; ou f) por medo de sentir-se mal, ter crise ou dor. Duas pacientes referiram que quando querem muito comer carne vermelha, alimento que para elas faz mal, apenas mastigam e jogam fora. Sentiam-se conformados com a restrição alimentar 36,9\% ( $\mathrm{n}=$ 24) dos participantes; $15,4 \%(\mathrm{n}=10)$ eram frustrados; $12,3 \%(\mathrm{n}=8)$ tristes ou indiferentes; $7,7 \%$ $(\mathrm{n}=5)$, confortáveis; $6,2 \%(\mathrm{n}=4)$, estressados; e para 9,2\% $(\mathrm{n}=6)$, não houve restrições. Quando relacionados os sentimentos gerados pela restrição alimentar com o tempo de acompanhamento psicológico, não houve associação estatisticamente significativa $(p=0,244)$.

Os pacientes com DC avaliados por Norton et al. ${ }^{20}$ também relataram o impacto da dieta sobre a vida diária, decorrente de mudá-la completamente, restringir ou evitar alimentos ou grupos alimentares e lidar com sintomas como dores no estômago por comer alimentos vistos como "proibidos".

\section{CONSIDERAÇÕES FINAIS}

As mudanças alimentares e psicossociais parecem iminentes ao diagnóstico de DII, já que a grande maioria dos pacientes restringe algum alimento e sofreu alguma modificação psicossocial em sua vida após o desenvolvimento da doença. Álcool, refrigerante, leite e derivados e alimentos gordurosos foram os alimentos/bebidas mais evitados pelos pacientes desta pesquisa. Além disso, a doença parece ter gerado impacto socioemocional e profissional em suas vidas. Diante do exposto, faz-se necessário um atendimento interdisciplinar, além de um olhar humanizado e integral por parte dos profissionais para esse paciente, que perpassa por modificações muito além das biológicas. No entanto, mais estudos são necessários para avaliar o impacto dessas mudanças após o desenvolvimento da DII.

\section{REFERÊNCIAS}

1. ACCIARI, A. S. et al. Effects of transpersonal brief psychotherapy on general state of health and quality of life in patients with Crohn's disease. J coloproctol (Rio J). v. 35, n. 3, p. 162-167, 2015.

2. ALONSO, E. C.; SAN ROMÁN, A. L.; MIQUEL, D. B. Impacto de la enfermedad inflamatoria intestinal sobre la función sexual. Gastroenterol Hepatol. v. 32, n. 2, p. 50-54, 2009. 
3. AÑEZ, M.; FUENMAYOR, M.; ROMERO, G. Enfermedad inflamatoria intestinal: rectocolitis úlcerosa idiopática y enfermedad de Crohn. Revista GEN. v. 66, n. 3, p. 197-206, 2012.

4. BACK, I. R. et al. Body composition in patients with Crohn's disease and ulcerative colitis. Arq Gastroenterol. v. 54, n. 2, p. 109-114, 2017.

5. BARROS, P. A. C.; SILVA, A. M. R.; LINS NETO, M. A. F. The epidemiological profile of inflammatory bowel disease patients on biologic therapy at a public hospital in Alagoas. J coloproctol (Rio J). v. 34, n. 3, p. 131-135, 2014.

6. BAUMGART, D. C. The diagnosis and treatment of Crohn's disease and ulcerative colitis. Dtsch Arztebl Int. v. 106, n. 8, p. 123-133, 2009.

7. BOUTROS, M.; MARON, D. Inflammatory bowel disease in the obese patient. Clin Colon Rectal Surg. v. 24, n. 4, p. 244-252, 2011.

8. COHEN, D.; BIN, C. M.; FAYH, A. P. T. Assessment of quality of life of patients with inflammatory bowel disease residing in southern Brazil. Arq Gastroenterol. v. 47, n. 3, p. 285-289, 2010.

9. FALCÃO, L. T. M.; MARTINELLI, V. F. Associação de doença inflamatória intestinal com ansiedade e depressão: avaliação dos fatores de risco. GED gastroenterol endosc dig. v. 35, n. 2, p. 52-58, 2016.

10. FARRUKH, A.; MAYBERRY, J. F. Inflammatory bowel disease in hispanic communities: a concerted south american approach could identify the etiology of Crohn's disease and ulcerative colitis. Arq Gastroenterol. v. 51, n. 3, p. 271-275, 2014.

11. FAUST, A. H. et al. Psychosocial factors contributing to inflammatory bowel disease activity and health-related quality of life. Gastroenterol Hepatol. v. 8, n. 3, p. 173-181, 2012.

12. FAVERI, A. et al. Hábitos de vida, perfil nutricional e consumo alimentar de pacientes com doença inflamatória intestinal atendidos ambulatorialmente. Rev Bras Nutr Clin. v. 28, n. 3, p. 188-191, 2013.

13. FERNANDES, L. L. et al. Cuidados alimentares nas doenças inflamatórias intestinais. Cad. Cult. Ciênc. v. 13, n. 1, p. 49-60, 2014.

14. GOUVEIA, E. C.; ÁVILA, L. A. Aspectos emocionais associados a disfunções gastroenterológicas. Psicol Estud. v. 15, n. 2, p. 265-273, 2010.

15. HARDT, M. R. et al. Epidemiological profile of 175 patients with Crohn's disease submitted to biological therapy. J Coloproctol. (Rio J). v. 32, n. 4, p. 395-401, 2012.

16. HARVEY, R. F.; BRADSHAW, J. M. A simple index of Crohn's-disease activity. Lancet. v. 315, p. $514,1980$.

17. KLEINUBING-JÚNIOR, H. et al. Perfil dos pacientes ambulatoriais com doenças inflamatórias intestinais. ABCD Arq Bras Cir Dig. v. 24, n. 3, p. 200-203, 2011.

18. LIMA, F. A. V. et al. Oscilação do humor em pacientes com doença de Crohn: incidência e fatores associados. Rev Assoc Med Bras. v. 58, n. 4, p. 481-488, 2012.

19. MARTÍNEZ GÓMEZ, M. J.; MELIÁN FERNÁNDEZ, C.; ROMEO DONLO, M. Nutrición en enfermedad inflamatoria intestinal. Nutr Hosp. v. 33, n. 4, p. 60-63, 2016.

20. NORTON, B. A. et al. Patient perspectives on the impact of Crohn's disease: results from group interviews. Patient Prefer Adher. v. 6, p. 509-520, 2012. 
21. NUTRITION SCREENING INITIATIVE. Incorporating nutrition screening and interventions into medical practice: a monograph for physicians. Washington, DC: American Academy of Family Physicians, The American Dietetic Association, National Councilon Aging Inc., 1994.

22. RIPOLI, J. et al. Nutritional follow-up of patients with ulcerative colitis during periods of intestinal inflammatory activity and remission. Arq Gastroenterol. v. 47, n. 1, p. 49-55, 2010.

23. RODRIGUES, S. C.; PASSONI, C. M. S.; PAGAnOTTO, M. Aspectos nutricionais na doença de Crohn. Cad. da Esc. de Saúde. v. 1, p. 1-8, 2008.

24. SAINSBURY, A.; HEATLEY, R. V. Review article: psychosocial factors in the quality of life of patients with inflammatory bowel disease. Aliment Pharmacol. v. 21, p. 499-508, 2005.

25. SAJADINEJAD, M.S. et al. Psychological issues in inflammatory bowel disease: an overview. Gastroenterol. Res. Pract. v. 2012, p. 1-11, 2012.

26. SCHROEDER, K. W.; TREMAINE, W. J.; ILSTRUP, D. M. Coated oral 5-aminosalcylic acid therapy for mildly to moderately active ulcerative colitis. N Engl J Med. v. 317, n. 26, p. 1625-1629, 1987.

27. SILVA, A. F. et al. Relação entre estado nutricional e atividade inflamatória em pacientes com doença inflamatória intestinal. ABCD Arq Bras Cir Dig. v. 23, n. 3, p. 154-158, 2010.

28. SILVA, A. F.; SCHIEFERDECKER, M. E. M.; AMARANTE, H. M. B. S. Ingestão alimentar em pacientes com doença inflamatória intestinal. ABCD Arq Bras Cir Dig. v. 24, n. 3, p. 204-209, 2011.

29. SOUZA, M. M. et al. Qualidade de vida de pacientes portadores de doença inflamatória intestinal. Acta Paul Enferm. v. 24, n. 4, p. 479-484, 2011.

30. TIMMER, A. et al. Determinants of female sexual function in inflammatory bowel disease: a survey based cross-sectional analysis. BMC Gastroenterol. v. 8, n. 45, p. 1-10, 2008.

31. WORLD HEALTH ORGANIZATION. Physical Status: the use and interpretation of anthropometry. Geneva, Switzerland: WHO, 1995. 